\title{
Optimization and in Vitro Evaluation of Injectable Sustained-Release of Levothyroxine Using PLGA-PEG-PLGA
}

\author{
Hossein Kamali ${ }^{1,2}$. Elham Khodaverdi ${ }^{1,2}$ Ehsan Kaffash ${ }^{1,2} \cdot$ Amir Sarem Saffari ${ }^{1,2}$. \\ Seyedeh Nesa Rezaeian Shiadeh ${ }^{1,2} \cdot$ Ali Nokhodchi $^{3}$ (D) Farzin Hadizadeh ${ }^{4,5}$
}

Published online: 11 August 2020

(C) The Author(s) 2020

\begin{abstract}
Purpose In situ-forming gels (semi-solid state) (ISFGs) are widely used as sustained drug delivery, but they show a high burst release as well. The purpose of the current study is to make triblock that can make a quick gel on injection with a minimum burst release.

Methods In this study, to control the release of levothyroxine from ISFG, PLGA-PEG-PLGA (triblock) polymer was used. The melting method was employed to synthesize the triblock via ring-opening polymerization (ROP). Different weight percentages of triblock in the formulation were investigated to reach the minimum initial burst release of levothyroxine from ISFGs. Furthermore, the results of the in-situ forming implant (solid-state) (ISFI) of levothyroxine prepared from PLGA $504 \mathrm{H}$ polymers were compared with ISFG.

Results The melting method employed in this study showed a successful ROP of the triblock. As the \% triblock concentration was increased from 30 to $50 \%$, the initial burst release decreased significantly. The initial burst release levothyroxine from ISFG $(6.52 \pm 0.30 \%)$ was much lower than the amount of levothyroxine released from ISFI $(14.15 \pm 0.79 \%)$. No cytotoxicity was observed for the sustained-release formulation containing ISFG 50\% according to the MTT assay.

Conclusion The results indicated that this formulation was safe to be administered subcutaneously. As the synthesized triblock has thermosensitive properties, and also has the hydrogen bonding between the N-methyl pyrrolidone molecules and PEG, therefore, these properties make ISFG formulation to have a smaller initial burst release compared to ISFI formulation.
\end{abstract}

Keywords Levothyroxine $\cdot$ Initial burst release $\cdot$ In situ-forming implant $\cdot$ In situ-forming gel

Ali Nokhodchi

a.nokhodchi@sussex.ac.uk

$\triangle$ Farzin Hadizadeh

hadizadehf@mums.ac.ir

1 Targeted Drug Delivery Research Center, Pharmaceutical Technology Institute, Mashhad University of Medical Sciences, Mashhad, Iran

2 Department of Pharmaceutics, School of Pharmacy, Mashhad University of Medical Sciences, Mashhad, Iran

3 Pharmaceutics Research Laboratory, School of Life Sciences, University of Sussex, Brighton, UK

4 Biotechnology Research Center, Pharmaceutical Technology Institute, Mashhad University of Medical Sciences, Mashhad, Iran

5 Department of Medicinal Chemistry, School of Pharmacy, Mashhad University of Medical Sciences, Mashhad, Iran

\section{Introduction}

In hypothyroidism disease, the primary function of the human thyroid, endogenous production of thyroxine (T4), and triiodothyronine (T3) is below normal. Enough secretion of these thyroid hormones is essential to maintain cardiovascular, physical, and mental health, as well as the growth and development of children which is done via the regulation of gene transcription $[1,2]$. Levothyroxine (T4), as a thyroidstimulating hormone, is primarily used in the treatment of hypothyroidism.

Levothyroxine sodium is administered orally as a thyroid hormone $[3,4]$. The oral bioavailability of levothyroxine is $65 \%$, which is achieved if consumed $30 \mathrm{~min}$ to $1 \mathrm{~h}$ before breakfast on an empty stomach with a full glass of water [5, 6] because of complex formation of levothyroxine with soybean products, sodium, calcium, and other minerals [7]. Furthermore, levothyroxine should be taken daily, and this 
requires the patient's full cooperation with physicians and pharmacists. Therefore, sustained-release formulation of levothyroxine can be a good choice in hypothyroidism for patients who have poor compliance [8].

Recently, in situ-forming implants (solid-state) (ISFI) are one of the popular sustained drug delivery systems containing poly D,L-lactic-co-glycolic acid (PLGA) as a water-insoluble biocompatible and biodegradable polymer, and N-methyl pyrrolidone (NMP) as a physiologically compatible and watermiscible organic solvent [9]. After injection of the formulation into an aqueous environment, the NMP diffuses into the release medium, while the water diffuses into the polymer matrix (phase inversion mechanism); hence, the polymer precipitates as soon as it contacts water leading to the formation in the solid polymeric implant $[10,11]$. NMP is an organic solvent and used in some of the products in the market such as Doxirobe ${ }^{\circledR}$ (doxycycline hyclate using PLA), Eligard ${ }^{\circledR}$ (leuprorelin acetate using PLGA) and Nuflor ${ }^{\circledR}$ (Florfenicol using PEG) $[12,13]$. However, the high-burst release in the first $24 \mathrm{~h}$ (15-80\% of the total drug contents) is a drawback of these kinds of ISFIs $[14,15]$. This initial burst release occurs by emerging NMP with the drug into the water upon injection that subsequently could cause systemic toxicity and tissue inflammation [16].

ISFI presents several significant advantages such as the avoidance of the first-pass metabolism, higher bioavailability for poorly water-soluble drugs, avoiding the escape of the treatment by the patients, which is especially relevant in indications where the lack of adherence to oral treatment may lead to incapacity and loss of autonomy [17-19]. Furthermore, ISFI utilizes bioresorbable polymers to control the drug release after parenteral administration. So, contrary to non-bioresorbable, releasing implants do not require surgical excision to remove the drug releasing system once the drug delivery is complete.

In this study, the use of triblock (PLGA-PEG-PLGA) instead of PLGA was suggested to prevent initial burst release. It seemed that the hydrogen bonding between the NMP molecules and PEG prevented the rapid diffusion of NMP into the release medium. Furthermore, the thermosensitive properties of PLGA-PEG-PLGA triblock could be helpful in matrix formation and decrease the initial burst release. Triblock should be liquid at room temperature and quickly convert into a gel at the physiological temperature $\left(37^{\circ} \mathrm{C}\right)[20,21]$. Therefore, there is no need for any surgery for the placement of this injectable triblock formulation [22]. The main drawback is that, generally, triblock is not stable in the presence of water. If water has to be used to make gels for mixing the triblock and drug powder (each in a separate vial), ultrasonication and a bath shaker are needed to be used for about $24 \mathrm{~h}$ to prepare the gel (semi-solid state). This makes it clinically impossible to use. To solve this problem, NMP was used to dissolve the triblock.
In the current research, to prepare ISFI formulations, only two syringes or vials were filled by the polymeric solution (polymer + NMP), and the drug in powder form is mixed together before injection and no need to use water (Fig. 1). The prepared formulation was administered at the injection site, and the gel is formed through two mechanisms: (a) phase inversion and (b) thermosensitive response of the triblock. The benefit of this formulation containing triblock is to have faster dissolution in NMP for preparing formulation and also making a quick gel on injection. Moreover, in the current study, to prevent the burst release of levothyroxine the application of the different weight ratios of triblock rather than PLGA is suggested. In situ-forming gel of levothyroxine has also been not formulated in other studies. It is hypothesized that the diffusion of NMP into the dissolution medium could be decreased by the hydrogen bonding between the PEG of triblock and NMP, and also with the thermo-sensitive characteristics of the triblock.

\section{Material and Methods}

\section{Materials}

For the ROP, PEG 1500, D,L-Lactide, glycolide, and stannous octoate ( $\left.\mathrm{Sn}(\mathrm{Oct})_{2}\right)$ were used (Merck, Germany). Levothyroxine sodium was supplied from Iran Hormone Pharmaceutical Co. (Iran). PLGA RG 504H (50:50, $\mathrm{M}_{\mathrm{w}}$ 38,000-54,000 Da) and levothyroxine were purchased from Sigma (USA) as the standard materials. 3-(4,5-dimethylthiazol-2-yl)-2, diphenyltetrazolium bromide (MTT) and Nmethyl pyrrolidone (NMP) were also provided by Merck, Germany. Roswell Park Memorial Institute (RPMI) 1640 culture medium, fetal bovine sera (FBS), penicillin-streptomycin, and trypsin were purchased from Gibco, Germany. Mouse fibroblast L929 cell lines were donated from the Pharmaceutical Technology Institute of Mashhad University of Medical Science (Iran).

\section{Methods}

\section{The Application of Melting Method (Three-Necked Flask) for the ROP of Triblock}

The melting method using a three-necked flask was employed to synthesize the triblock copolymer. The ROP scheme was shown in Fig. 2a [23, 24]. Five grams of PEG was loaded into a vacuum three-necked flask under continuous stirring using a magnetic stirrer for $0.5 \mathrm{~h}$ to evaporate the absorbed water $(T=$ $\left.100{ }^{\circ} \mathrm{C}\right)$ from PEG. Next, the catalyst Sn $(\mathrm{Oct})_{2}(20 \mu \mathrm{L})$, D,Llactide $(9.45 \mathrm{~g})$, and glycolide $(2.54 \mathrm{~g})$ (LA:GA molar ratio (3:1)) were added into the polymeric solution under vacuum condition for $24 \mathrm{~h}\left(T=140{ }^{\circ} \mathrm{C}\right)$. After the completion of 
Fig. 1 Preparation of ISFI or ISFG using two coupled syringes [21]
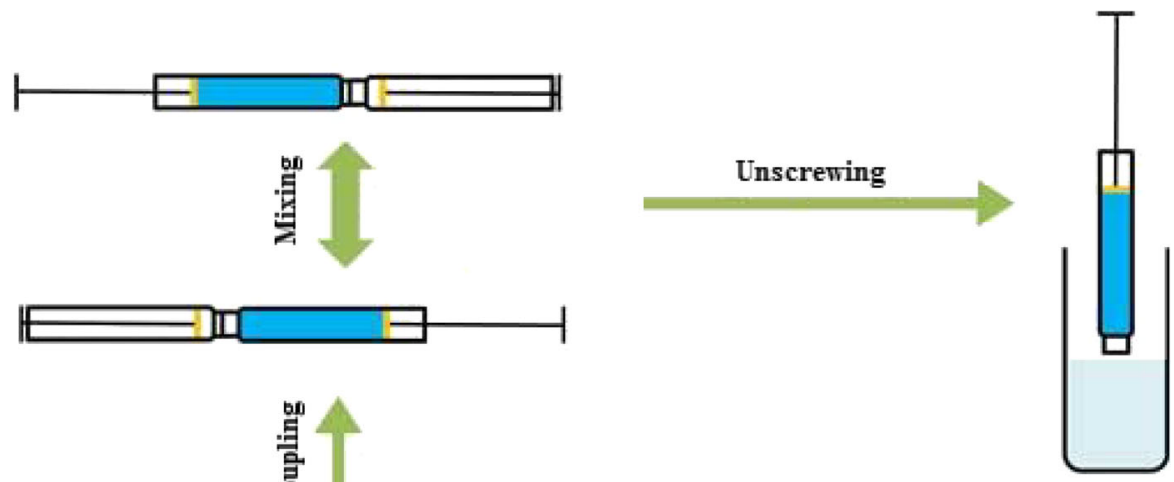

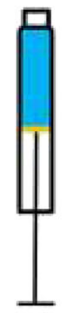

Syringe for polymeric solution (polymer + NMP+ additive)

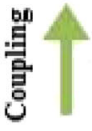

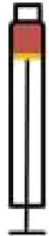

Syringe for drug powder
Implant or Composite polymerization, the copolymer was dissolved in water (5$8{ }^{\circ} \mathrm{C}$ ) and precipitated at $60^{\circ} \mathrm{C}$ for purification. The unreacted monomer, low-molecular-weight, and water-soluble polymer in the supernatant were eliminated. Finally, the precipitate was collected and freeze-dried at a temperature of $-20{ }^{\circ} \mathrm{C}$ at a pressure of 103.2446 $\mathrm{Pa}$ to remove the residual water [25, 26]. The purification process was repeated three times to obtain the purified triblock [27].

\section{Characterization of Triblock}

${ }^{1} \mathrm{H}-\mathrm{NMR}$ To evaluate the structure of triblock (PLGA-PEGPLGA) and LA:GA molar ratio of the triblock copolymer, the ${ }^{1} \mathrm{H}-\mathrm{NMR}$ spectrum of the copolymer was obtained by Bruker FT-500 and $300 \mathrm{MHz}$ instruments (USA) in $\mathrm{CDCL}_{3}$ at room temperature $[28,29]$. The LA:GA ratio was determined by integration of the signals pertaining to each

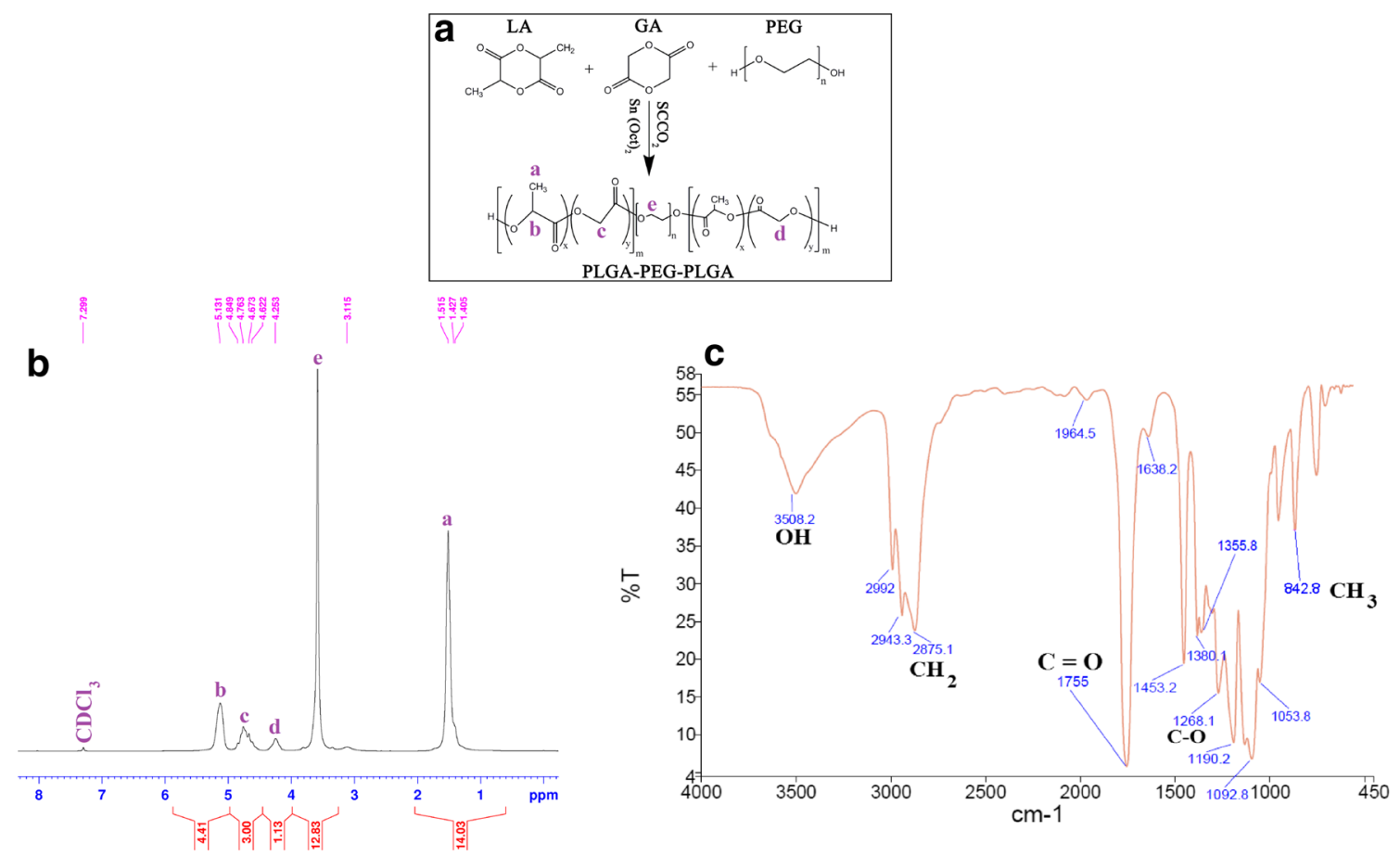

Fig. 2 ROP scheme (a) [21], ${ }^{1}$ H-NMR (B), and FTIR spectrum (c) of triblock 
monomer, such as the peaks from the $\mathrm{CH}$ and $\mathrm{CH} 3$ groups of LA and the $\mathrm{CH} 2$ groups of GA.

Fourier Transform Infrared Analysis The triblock was mixed with $\mathrm{KBr}$, and then its structure was also confirmed by the Fourier transform infrared analysis (FTIR) (vertex 70, Bruker, Germany) [23, 30].

Polydispersity Index To determine the polydispersity index (PDI), average molecular weight $\left(M_{w}\right)$, and the number average molecular weight $\left(M_{n}\right)$ of the synthesized triblock, a GPCAddon apparatus with Plgel $®$ columns (Agilent, USA) was used. In the GPC technique, tetrahydrofuran as eluent at a flow rate of $1 \mathrm{~mL} / \mathrm{min}$ was employed, and polystyrene was used as a standard [23].

Differential Scanning Calorimetry To identify the glass transition and melting temperatures of triblock, differential scanning calorimetry (DSC) was conducted by a DSC 822 (Mettler Toledo, Switzerland). Approximately, a few mg of samples (3-4 mg) were accurately weighed into aluminum pans and then sealed. The pans were cooled down to $60{ }^{\circ} \mathrm{C}$ followed by heating the samples to $60^{\circ} \mathrm{C}$ at a scanning rate of $5{ }^{\circ} \mathrm{C} / \mathrm{min}$ under nitrogen atmosphere.

Sol-Gel Transition Temperature To determine the sol-gel transition temperature of the synthesized triblock, different weight $\%$ of triblock ranging from 10 to $50 \mathrm{w} / \mathrm{w} \%$ was prepared in PBS (pH 7.4), and the temperature of the prepared samples was elevated from 0 to $60^{\circ} \mathrm{C}$ at a scanning rate of $0.5^{\circ} \mathrm{C} / \mathrm{min}$. The sol-gel transition temperature was recorded when the magnetic flea in the solution stopped spinning [31,32].

Scanning Electron Microscopy To evaluate cross-sections morphology of the matrix, the gel which formed after 3 days was freeze-dried and analyzed using SEM (LEO1450 vp, Zeiss Company, Germany) [24]. Scanning electron microscopy (SEM, Tescan, model Vega II, Czech) was equipped with an energy dispersive X-ray analyzer system (EDXA, Oxford Instrument, INCA, England). Samples were freeze-dried and placed on the specimen holder using double-sided carbon conductive tape and coated with gold to render them electrically conductive.

\section{Preparation of Liquid Formulations}

PLGA 504H (ISFI, 33\% w/w) and the triblock (ISFG, 30, 40, and $50 \% \mathrm{w} / \mathrm{w}$ ) were dissolved in NMP and sonicated at room temperature for $2 \mathrm{~h}[33,34]$. To sterilize the formulations, an autoclave was used for $15 \mathrm{~min}$ at $121^{\circ} \mathrm{C}$ at 3 bars. To complete the formulation, $3 \mathrm{mg}$ of levothyroxine sodium was added to the autoclaved polymeric solution followed by sonication of the sample to achieve a homogeneous solution. The formulations were passed through a 20 -gauge syringe at $25^{\circ} \mathrm{C}$ at a steady shear rate of $75 \mathrm{~s}^{-1}$ to investigate the syringability of each sample. A cone/plate viscometer (Brookfield, Germany) at a shear rate of 0.3 r.p.m. was used to measure the viscosities of ISFG formulations [35].

\section{In Vitro Release Study}

To obtain dissolution profiles for the prepared formulations, the dissolution test was performed. Phosphate buffer with $\mathrm{pH} 7.4(50 \mathrm{~mL})$ was used as a dissolution medium, and the medium was kept at $37 \pm 0.5{ }^{\circ} \mathrm{C}$ with a rotational speed of $35 \mathrm{rpm}$. One milliliter of the formulation was injected directly into each vial containing dissolution media (100 mL PBS) by a 20 -gauge needle. The solution turned into a gel or an implant after injection to the medium. The vials were kept in a reciprocal shaking water bath during the entire release study. The test was followed by withdrawing manually $3 \mathrm{~mL}$ from the dissolution medium at predetermined time intervals up to 35 days $(2,4,6,8,12,18$, and $24 \mathrm{~h} ; 2,3,4,5,7,10,14,16$, $18,21,28$, and 35 days). Following the withdrawal of the samples, $3 \mathrm{~mL}$ of fresh PBS was transferred to the dissolution medium to keep the volume of the dissolution medium constant, and also to maintain a sink condition $[36,37]$. The withdrawn samples were subsequently analyzed by HPLC to determine the concentrations of levothyroxine from the calibration curve $(0.007-10 \mu \mathrm{g} / \mathrm{mL})$. The samples $(20 \mu \mathrm{L})$ were analyzed through HPLC (Shimadzu, Japan) with an LC-6 AD pump linked to a diode array detector (DAD) at $225 \mathrm{~nm}$ with a C18 column $(25 \mathrm{~cm} \times 4.6 \mathrm{~mm}$, pore size $100 \AA)$. A mixture of double-distilled water $(59.5 \% \mathrm{v} / \mathrm{v})$, acetonitrile $(40 \% \mathrm{v} / \mathrm{v})$, phosphoric acid $(0.5 \% \mathrm{v} / \mathrm{v})$ (isocratic) with a flow rate of $1.2 \mathrm{~mL} / \mathrm{min}$ was used as eluent. The NMP was also detected in the release medium at $220 \mathrm{~nm}$ with double-distilled water $(68 \% \mathrm{v} / \mathrm{v})$ containing trifluoroacetic acid $(0.1 \% \mathrm{v} / \mathrm{v})$ and acetonitrile $(32 \% \mathrm{v} / \mathrm{v})$ (isocratic) as eluent with a flow rate $0.5 \mathrm{~mL} / \mathrm{min}[38,39]$.

The weight of remained matrix (implant or gel) was determined at 1, 2, 3, 5, 7, 14, 21, 28, and 35 days in the release medium (deionized water) after freeze-drying to monitor its degradation percentage using Eq. 1.

Degradation $\%=\frac{\left(W_{0}-W_{d}\right)}{W_{0}} \times 100$

where $w_{\mathrm{o}}$ and $w_{d}$ are the initial and secondary weight of gel or implant.

\section{In Vitro Cellular Cytotoxicity}

The RPMI 1640 medium supplemented with $10 \%$ (v/v) heatinactivated FBS, $100 \mathrm{IU} / \mathrm{mL}$ of penicillin, and $100 \mathrm{mg} / \mathrm{mL}$ of streptomycin containing the mouse L929 fibroblast cell line 
Fig. 3 GPC chromatogram of triblock

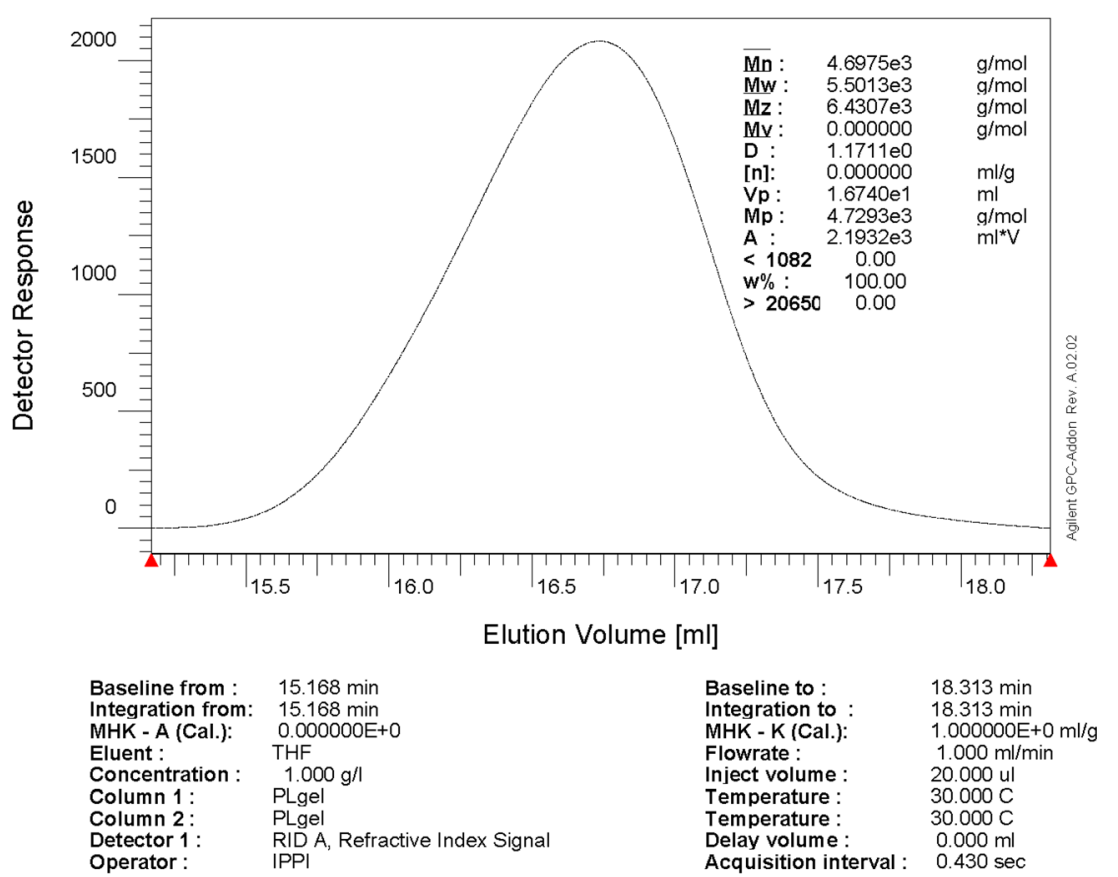

was incubated in a humidified incubator at $5 \% \mathrm{CO}_{2}$ at $37{ }^{\circ} \mathrm{C}$ for 1 week [11]. The cells were seeded at a 96-well plate at a density of $5 \times 10^{4}$ cells/well. The plates were divided into 6 groups ( $n=3$ per group): group I: control (containing cells and culture medium without sample); group II: levothyroxine solution in NMP (3 mg levothyroxine in $670 \mathrm{mg}$ NMP); group III: ISFI; group IV: ISFG 30\%; group VI: ISFG 40\%; and group VII: ISFG 50\%. Firstly, a $50 \mathrm{~mL}$ PBS (pH 7.4, $37^{\circ} \mathrm{C}$ ) for each group was prepared and incubated for $24 \mathrm{~h}$. Then, a sterile membrane (pore size $0.22 \mu \mathrm{m}$ ) was employed to filter $2 \mathrm{~mL}$ of this solution, and subsequently, $20 \mu \mathrm{L}$ of the filtered solution was directly added into the center of each well and incubated for $24 \mathrm{~h}$. Then, the culture medium was discarded, and the cell viability $\%$ was determined by adding $500 \mu \mathrm{L}$ of MTT solution $(0.5 \mathrm{mg} / \mathrm{mL}$ in PBS). After $4 \mathrm{~h}, 100 \mu \mathrm{L}$ of DMSO was replaced with the culture medium, then the plate

Fig. 4 DSC spectrum of triblock

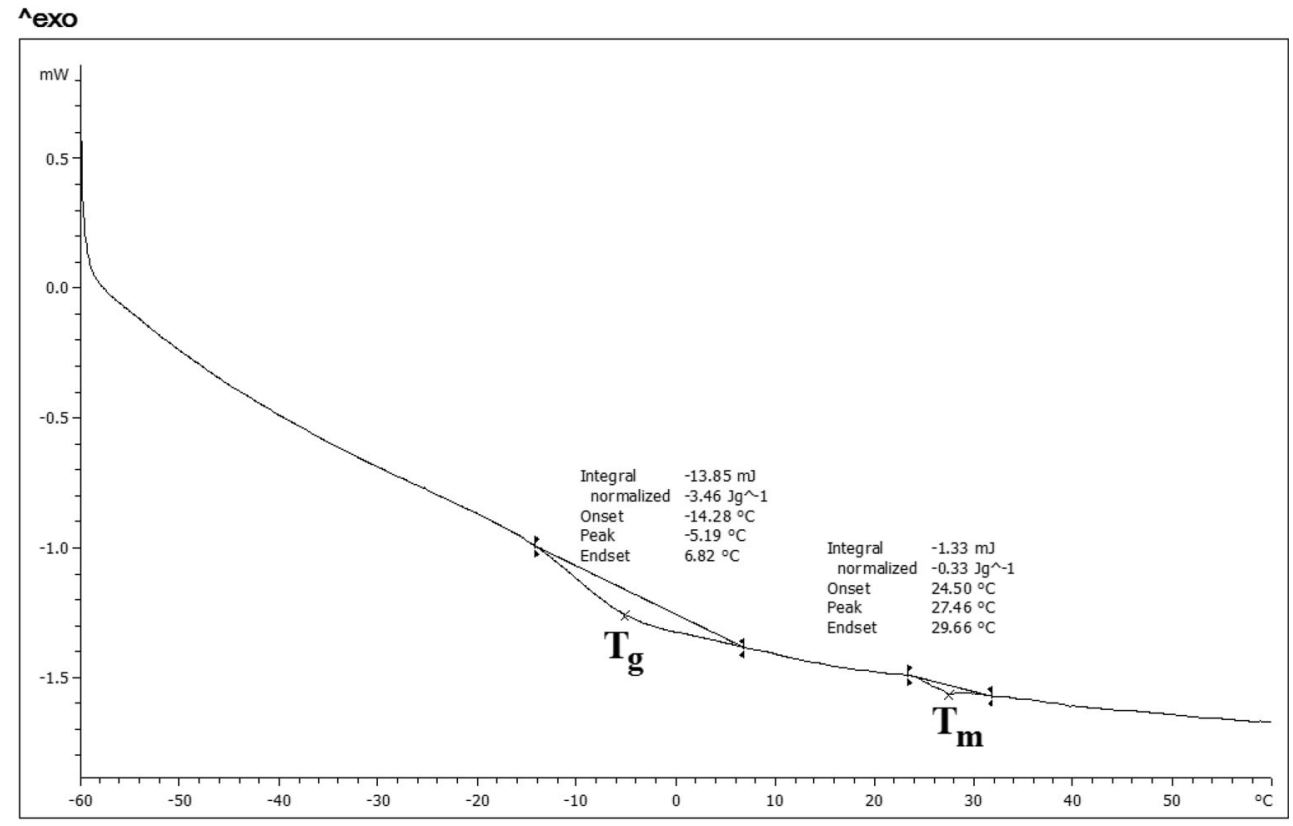

Lab: METTLER

STAR $^{\mathrm{e}}$ SW 12.00 


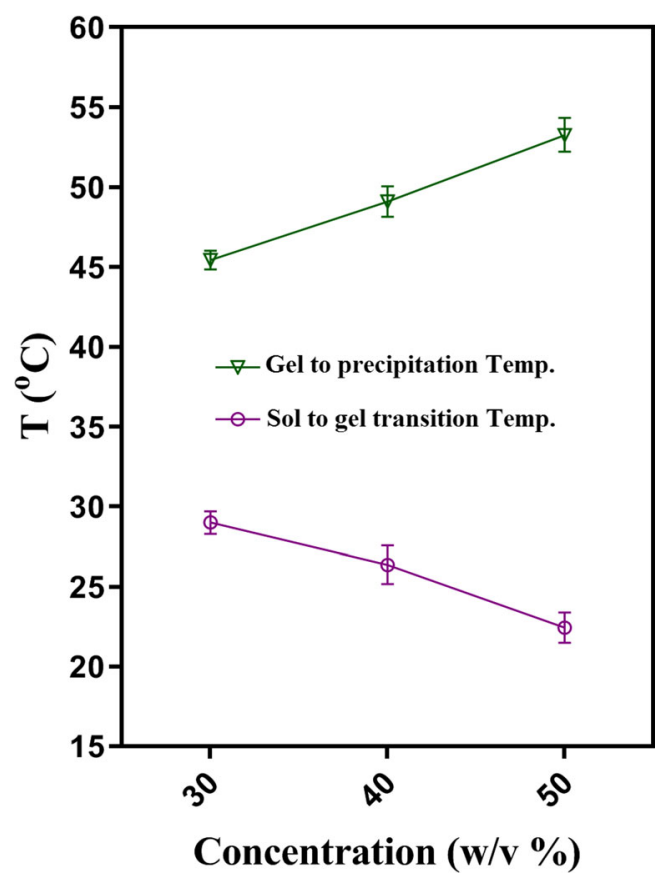

Fig. 5 Phase diagram of triblock (Sol to gel and gel to precipitate) (Mean $\pm \mathrm{SD}, n=3$ )

was agitated for $1 \mathrm{~h}$. Finally, the absorbance was measured by a microplate reader (VMax®, Canada) at $570 \mathrm{~nm}$ (sample) and a reference wavelength was set up at $630 \mathrm{~nm}$ [39].

\section{Statistical Analysis}

Data reported in the current research were expressed as means and standard deviations. To show the significance of the data, one-way ANOVA was performed followed by $t$ test using linear regression where $p<0.05$ was quoted as significant.

\section{Results and Discussion}

\section{ROP and Characterization of Triblock Copolymer}

In this study, the melting method was successfully employed to ROP of the triblock. A typical ${ }^{1} \mathrm{H}-\mathrm{NMR}$ spectrum of the triblock was shown in Fig. 2b. The formation of a terminal $\mathrm{CH}_{2}$ of PEG, the $\mathrm{CH}$ of LA, the $\mathrm{CH}_{3}$ of LA, and the $\mathrm{CH}_{2}$ of GA were identified by signals appearing at 4.253, 3.115, $5.131,1.427$, and 4.763 ppm, respectively. The structure of copolymer was also investigated using FTIR spectroscopy [40]. The FTIR characteristic peaks appearing at 3508.2, $1268.1,2875.1,1755$, and $842.8 \mathrm{~cm}^{-1}$ represented the terminal $\mathrm{OH}$ of PEG, C-O of PEG, $\mathrm{CH}_{2}$ of GA, and PEG, C=O of LA and GA and the $\mathrm{CH}_{3}$ of LA, respectively [41] (Fig. 2c). The results obtained by FTIR and 1H-NMR were in agreement with each other. The unimodal trace obtained by GPC and also a low polydispersity observed in GPC chromatogram of triblock were an indication of a successful synthesis and purification of the triblock (Fig. 3).

According to the DSC thermogram shown in Fig. 4, the melting temperatures $\left(T_{m}\right)$ and the glass transition temperature $\left(T_{g}\right)$ of triblock were about 27.46 and $-5.19^{\circ} \mathrm{C}$, respectively. The results indicated that the state of the synthesized triblock was semisolid. The phase diagram of triblock with different concentrations of copolymers was shown in Fig. 5. The results
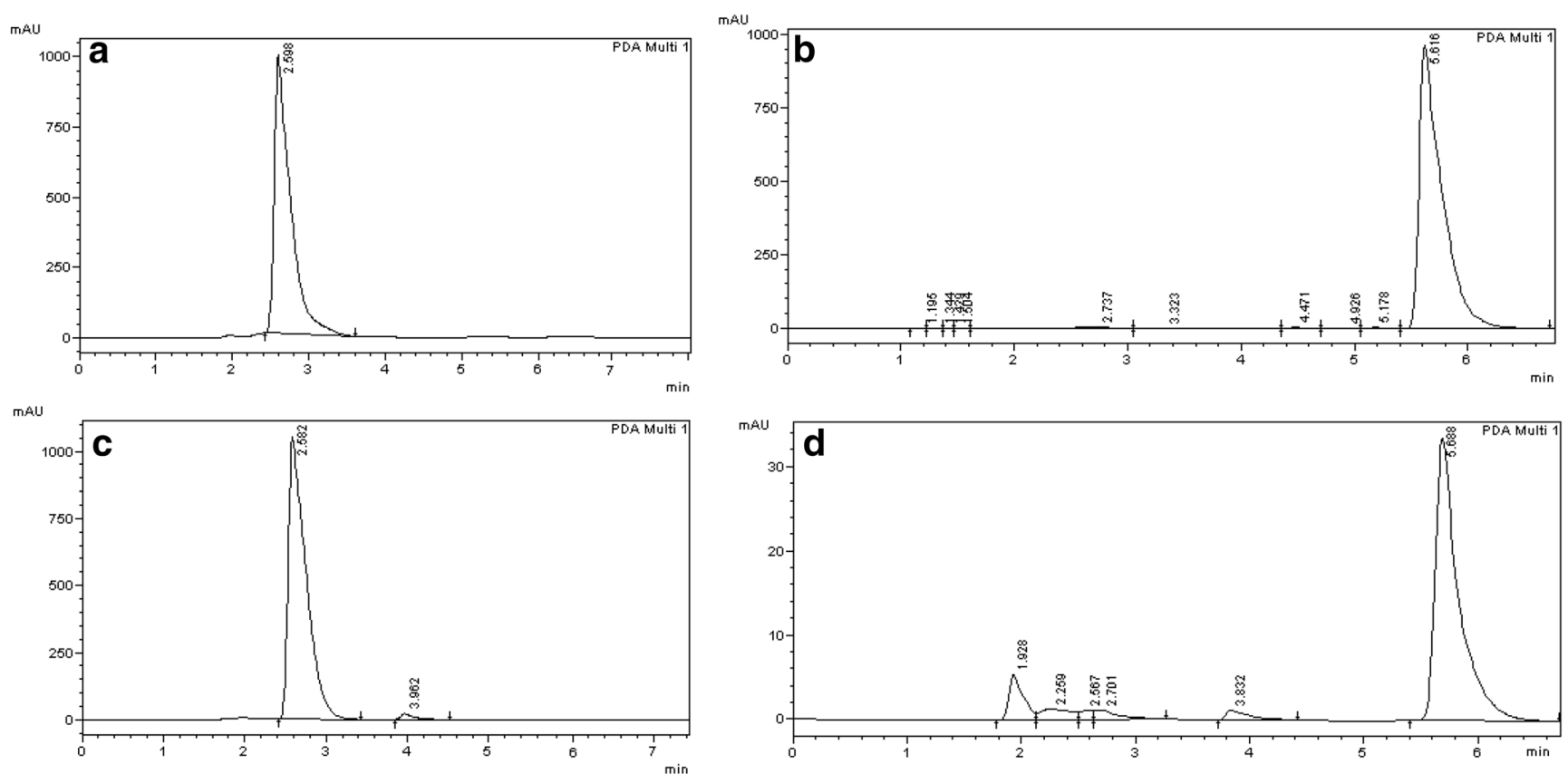

Fig. 6 Chromatogram of HPLC analysis for pure levothyroxine (a) and NMP (b) in the PBS $(10 \mu \mathrm{g} / \mathrm{mL})$, a sample of in vitro release medium from ISFG formulation after $24 \mathrm{~h}$ for levothyroxine (c) and NMP (d) 
presented in Fig. 5 exhibited that an increase in the concentration of the copolymer from 30 to $50 \%(\mathrm{w} / \mathrm{v})$ resulted in an increase in the precipitation temperature and also caused a reduction in the sol to gel transition temperature. The aggregation of the micelles was accelerated when the concentration of the copolymer was increased, this, in turn, could cause an enhancement in the micelle concentrations, and therefore, the transition of sol to gel occurred at a lower temperature, and the precipitation temperature decreased.

\section{In Vitro Release Evaluation}

HPLC chromatograms of pure levothyroxine, NMP in PBS, and levothyroxine and NMP for the ISFG formulation in PBS at $37^{\circ} \mathrm{C}$ after $24 \mathrm{~h}$ were shown in Fig. $6 \mathrm{a}-\mathrm{d}$, respectively. The HPLC thermograms showed retention times of $2.56 \pm$ $0.11 \mathrm{~min}$ and $5.62 \pm 0.28 \mathrm{~min}$ for levothyroxine and NMP, respectively. Sharp peaks from levothyroxine and NMP showed that the HPLC was able to separate the two substances from the release medium and be detectable. Also, the limit of quantification (LOQ) and the limit of detection (LOD) were $15.9 \mathrm{ng} / \mathrm{mL}$ and $5.3 \mathrm{ng} / \mathrm{mL}$ for levothyroxine and $3.9 \mu \mathrm{g} / \mathrm{mL}$ and $1.3 \mu \mathrm{g} / \mathrm{mL}$ for NMP respectively.

It was observed that all formulations were easily injectable through a 20 -gauge syringe at $25{ }^{\circ} \mathrm{C}$. Figure 7 showed the viscosity of formulations and the effects of triblock concentration in NMP on the viscosity [42]. The figure showed a nonlinear increase in the viscosity of PLGA-PEG-PLGA polymer solution in NMP with a sharp change at the concentration of $50 \%$ which could be due to more hydrophobic lactate concentration leading to the aggregation of triblock.

The gel or implant was formed immediately after the injection of the formulation into the release medium. The cumulative in vitro release of levothyroxine and NMP from ISFG (using different concentrations of triblock \%) and ISFI using PLGA were shown in Fig. 8a, b, respectively. The initial burst release of levothyroxine from ISFG $30 \%$, ISFG $40 \%$, and ISFG $50 \%$ were $17.30 \pm 0.90 \%, 9.57 \pm 0.28 \%$, and $6.52 \pm$

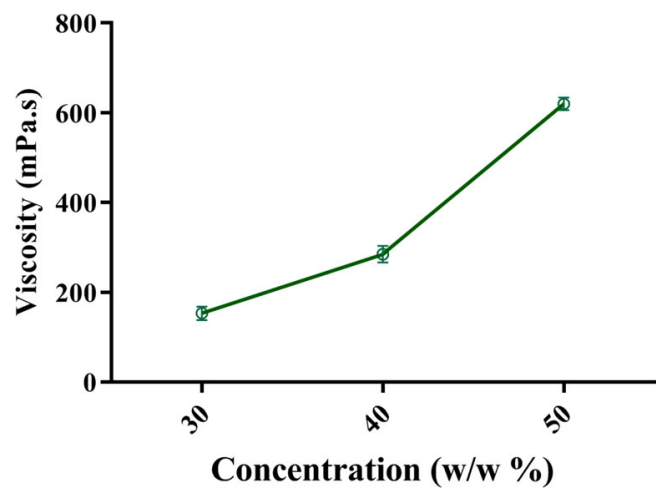

Fig. 7 The effect of viscosity vs. concentration of triblock solution in the NMP
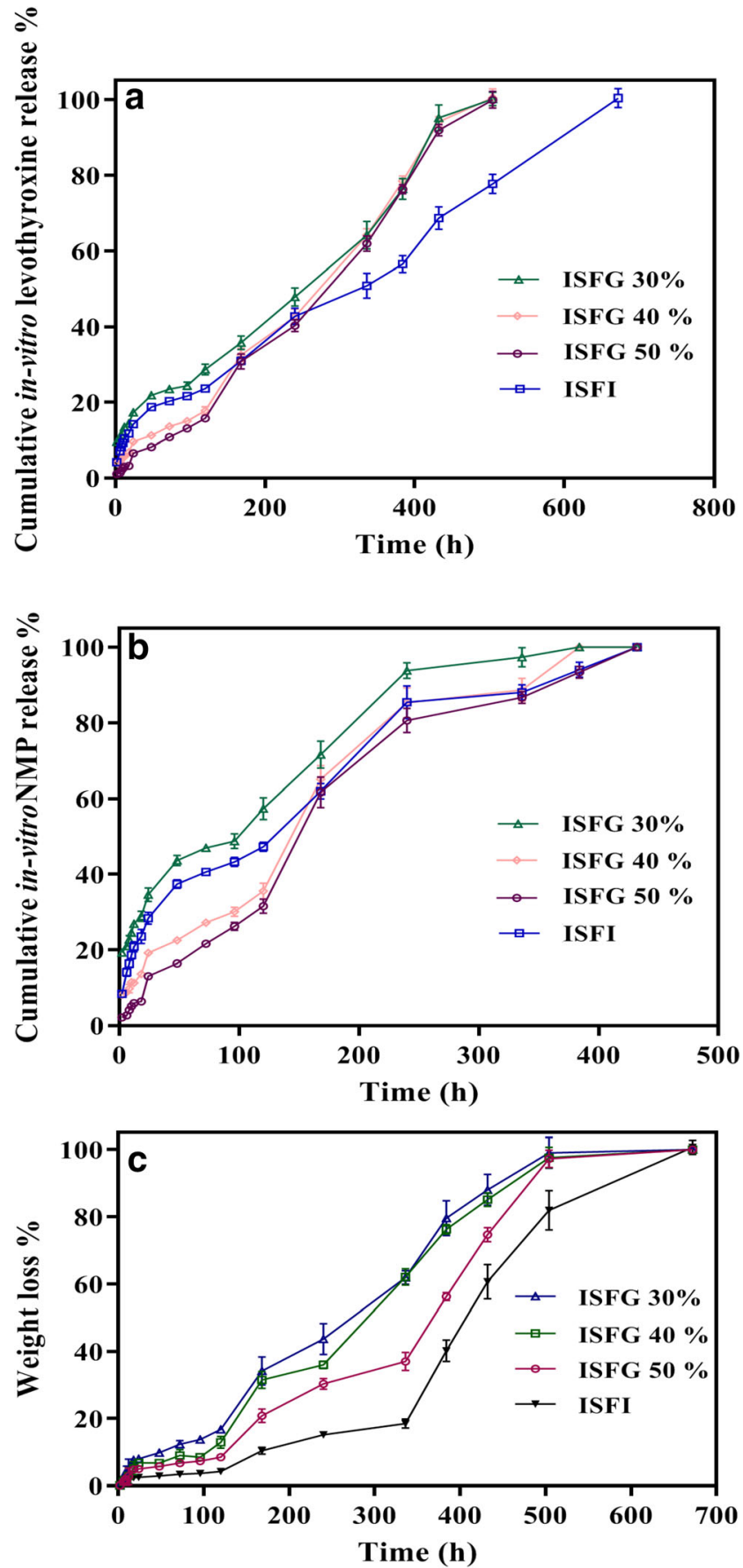

Fig. 8 Cumulative in vitro release of levothyroxine from formulations (a), cumulative in vitro release of NMP from formulations (b), and in vitro degradation of formulations without levothyroxine (c). (Mean \pm $\mathrm{SD}, n=3)$

$0.30 \%$, respectively. Furthermore, the initial burst release of NMP from ISFG 30\%, ISFG 40\%, and ISFG50\% were 34.06 $\pm 1.81 \%, 19.15 \pm 0.57 \%$, and $13.05 \pm 0.59 \%$, respectively. An increase in weight percentage of triblock from 30 to $50 \%$ caused an increase in the cross-links between the copolymer molecules which led to an increase in the tortuosity and the viscosity of the hydrogel. Subsequently, the initial burst 
a

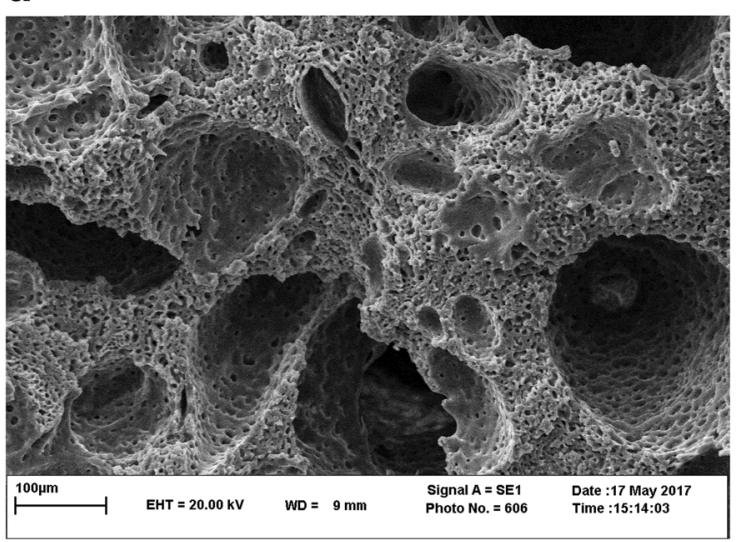

C

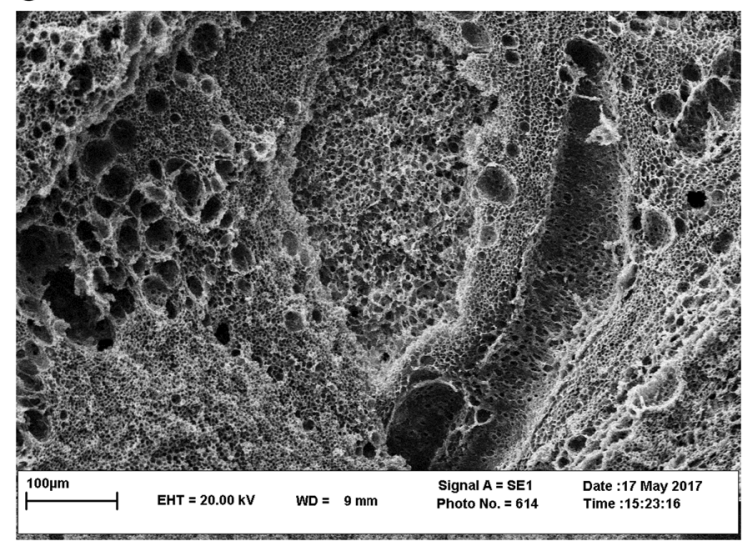

b

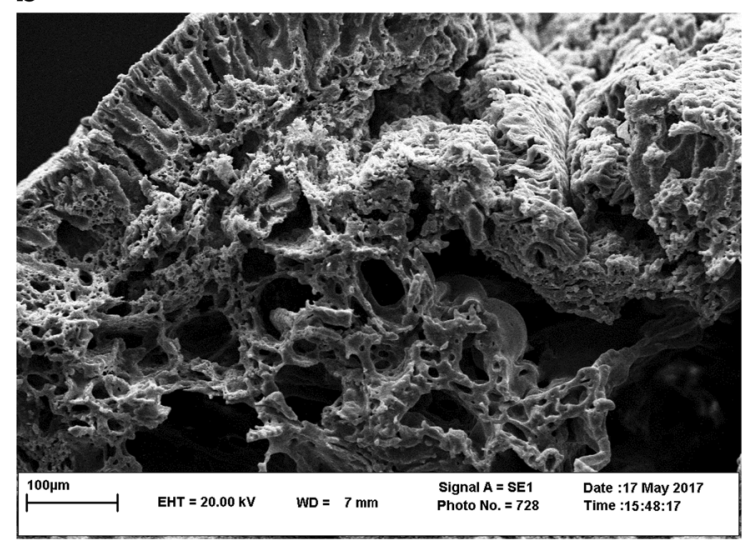

d

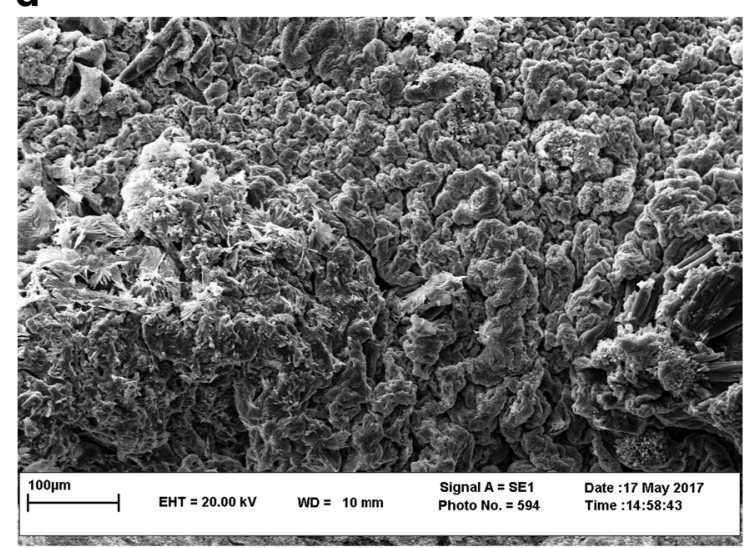

Fig. 9 Cross-section morphologies of the implant or gel from the formulations using SEM (scale bar: $100 \mu \mathrm{m}$ ): ISFI (a), ISFG 30\% (b), ISFG 40\% (c), and ISFG $50 \%$ (d)

release significantly reduced as a result of the increased in hydrogel network porosity, and a reduction in drug and NMP diffusion rate $[21,23,43]$. The early burst release of levothyroxine from the ISFI formulation $(14.15 \pm 0.79 \%)$ was significantly $(p<0.05)$ higher than the ISFG $50 \%(6.52$ $\pm 0.30 \%)$. Furthermore, NMP showed an early burst release of $28.31 \%$ from the ISFI formulation which was remarkably higher than the ISFG 50\% which showed $13.05 \%$ initial drug release $(p<0.05)$. A lower burst release of NMP observed for the ISFG formulation compared to ISFI could be related to the formation of hydrogen bonding between terminal hydroxyl groups of triblock and NMPs carbonyl group.

Based on the loss of weight, in vitro degradation results for ISFI and ISFG formulations without levothyroxine in the PBS were shown in Fig. 8c. The faster degradation rate of ISFG compared to ISFI formulation could be attributed to the existence of PEG in the triblock structure which caused an increase in the penetration of more water into the matrix [44, 45]. The faster degradation of triblock in comparison to PLGA $504 \mathrm{H}$ caused a complete drug release from the ISFG within

Table 1 Regression coefficient $\left(R^{2}\right)$ and " $n$ " of predicted in vitro release models

\begin{tabular}{|c|c|c|c|c|c|c|c|c|c|}
\hline \multirow[t]{2}{*}{ Model } & \multirow[b]{2}{*}{ Equations } & \multicolumn{2}{|c|}{ ISFG $30 \%$} & \multicolumn{2}{|c|}{ ISFG $40 \%$} & \multicolumn{2}{|c|}{ ISFG $50 \%$} & \multicolumn{2}{|l|}{ ISFI } \\
\hline & & $R^{2}$ & $n$ & $R^{2}$ & $n$ & $R^{2}$ & $n$ & $R^{2}$ & $n$ \\
\hline Higuchi (diffusion) & $Q_{t}=K_{H} \times \sqrt{ } t$ & 0.9108 & - & 0.8430 & - & 0.8178 & - & 0.932 & - \\
\hline Zero-order (degradation) & $Q_{t}-Q_{\mathrm{o}}=K_{o} \times \mathrm{t}$ & 0.9228 & - & 0.9854 & - & 0.9872 & - & 0.939 & - \\
\hline Korsmeyer-Peppas & $M_{t} / M_{\infty}=\mathrm{Kt}^{n}$ & 0.952 & 0.726 & 0.9867 & 1.075 & 0.9940 & 1.178 & 0.974 & 0.721 \\
\hline
\end{tabular}

$Q_{t}$, drug release at time $t ; Q_{0}$, the initial drug at time zero; $K_{0}$, zero-order constant; $K_{H}$, Higuchi constant; $M_{t}$, the drug released at time $t ; M_{\infty}$, the drug release at $t$, infinite; $k$, power-law constant; $n$, diffusion exponent [Fickian diffusion (less than 0.43), non-Fickian (between 0.43-0.89), zero-order (greater than 0.89)] 
21 days, while the complete drug release occurred for ISFI after 28 days. The in vitro degradation results and NMP release profiles both were confirmed the in vitro drug release results (Fig. 8a-c).

The morphology of ISFG with different weight $\%$ of triblock (ranging from 30 to $50 \mathrm{w} / \mathrm{w} \%$ ) in NMP and ISFI formulations were shown in Fig. 9. The images showed that ISFI formulation was spongy and highly porous (Fig. 9a), whereas the ISFG gels containing 30\% (Fig. 9b), 40\% (Fig. 9c), and $50 \%$ (Fig. 9d) of triblock showed low porosity and more packed morphology due to the decreased rate of NMP exchange with water that subsequently prevented the formation of a highly porous, spongy, and finger-like structure [46].

To evaluate the kinetics and mechanisms of drug release, the release data were fitted into various release models [47, 48]. The models used were zero-order, Higuchi, and Korsmeyer-Peppas (Ritger-Peppas) models which were listed in Table 1.

Table 1 shows the modeling of cumulative in vitro release experimental data based on zero-order, Higuchi, and Korsmeyer-Peppas models. The determination coefficient $\left(R^{2}\right)$ of the zero-order model was higher than the Higuchi model for four formulations (ISFG 30\%, 40\%, 50\%, and ISFI), which indicated that the release data followed well the zero-order model (Table 1). The result indicated that the main mechanism of the drug release followed the zero-order model (degradation). Furthermore, according to the KorsmeyerPeppaspas model, the $n$ value for ISFG $40 \%$ and ISFG 50\% was greater than 0.43 which indicated the drug release mechanism for these two formulations was also the degradation model. The $n$ value for ISFG $30 \%$ and ISFI was $0.43 \leq n<$ 0.89 which represented a non-Fickian transport. But, since the determination coefficient $\left(R^{2}\right)$ for the zero-order model of ISFG $30 \%$ and ISFI was greater than the Higuchi model, this indicated that the experimental data followed the zero-order model (Table 1). Therefore, the modeling of four formulations proved that the dominant mechanism of drug release was degradation (zero-order model) [49].

\section{In Vitro Compatibility Evaluation}

For any subcutaneously administered formulation, it is vital to investigate its side effects on the skin [50]. To this end, the biocompatibility projections of ISFI formulation including their cytotoxic effects were carried out via mouse L929 cell line (fibroblast cells) that has been initiated from adipose tissue with fibroblast morphology $[11,39]$. The cell viability after $24 \mathrm{~h}$ was investigated by employing MTT assay [51] and the results were shown in Fig. 10. Rahimi et al. evaluated the cytotoxicity effects of ISFI formulation on fibroblast cells after $24 \mathrm{~h}$ and reported cell viability values from 89 to $100 \%$ [39]. The cell viability of the ISFG $50 \%(96.17 \pm 1.15 \%)$ was higher than that of the ISFG $30 \%$ ( $86.37 \pm 0.76)$, ISFG $40 \%$

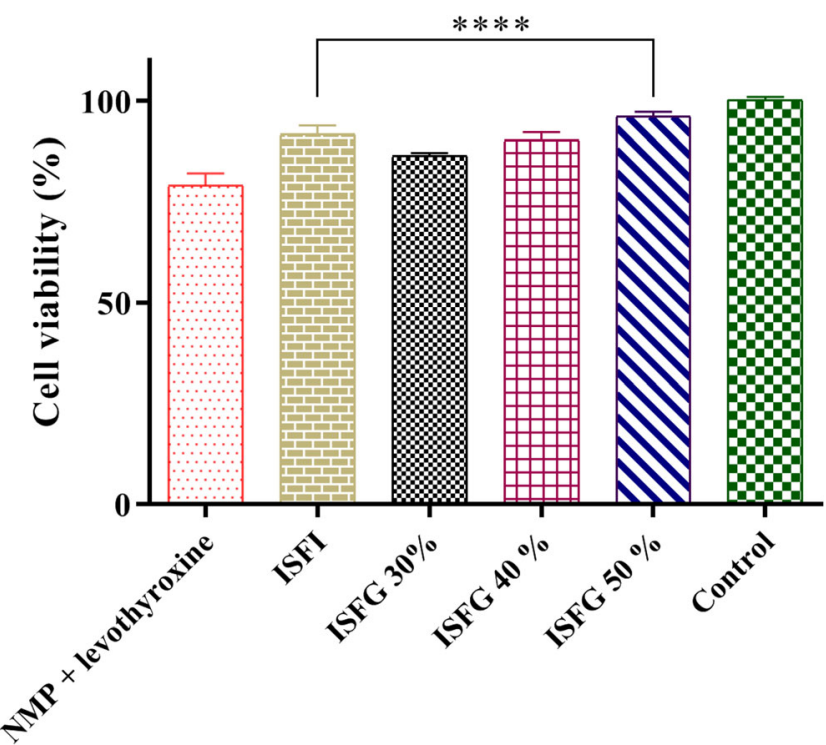

Fig. 10 Cell viability of L929 mouse fibroblast cells in contact with release media after $24 \mathrm{~h}$ using MTT assay. (mean $\pm \mathrm{SD}, n=3$ ). $* * * * p<0.0001$

$(90.27 \pm 2.01)$, and ISFI $(91.70 \pm 2.17 \%)$ as shown in Fig. 10 . The higher cell viability could be due to the lower early release of NMP and levothyroxine from this formulation which was confirmed by the in vitro release evaluation. Based on these results, it can be concluded that the formulation containing ISFG 50\% showed good biocompatibility; therefore, it could be administered subcutaneously.

\section{Conclusion}

One of the main problems of sustained-release formulations is the initial burst release of a drug at the beginning of the therapy during the first $24 \mathrm{~h}$. For levothyroxine depot forms, the high initial drug release increases the serum concentration of the drug which may cause serious side effects such as tachycardia and fatigue. In this work, PLGA-PEG-PLGA copolymer was used for ISFG preparation to overcome this phenomenon (controlling the early drug release), because the hydrogen bonding between the NMP molecules and the PEG chains in ISFG formulation prevented the rapid diffusion of NMP into the release medium and showed a lower initial drug release. Our results were indicated that the copolymer and solvent NMP were biodegradable, biocompatible, and enable to deliver the drug for a long time (21 days in vitro drug release) with the acceptable unwanted initial release than ISFI using PLGA 504H which has not yet been reported in other studies so far.

Funding Information This study was funded by the Vice Chancellor for Research and Technology of Mashhad University of Medical Sciences, Mashhad, Iran (project code: N-961554). 


\section{Compliance with Ethical Standards}

Conflict of Interest The authors declare that they have no conflict of interest.

Open Access This article is licensed under a Creative Commons Attribution 4.0 International License, which permits use, sharing, adaptation, distribution and reproduction in any medium or format, as long as you give appropriate credit to the original author(s) and the source, provide a link to the Creative Commons licence, and indicate if changes were made. The images or other third party material in this article are included in the article's Creative Commons licence, unless indicated otherwise in a credit line to the material. If material is not included in the article's Creative Commons licence and your intended use is not permitted by statutory regulation or exceeds the permitted use, you will need to obtain permission directly from the copyright holder. To view a copy of this licence, visit http://creativecommons.org/licenses/by/4.0/.

\section{References}

1. Tanguay M, Girard J, Scarsi C, Mautone G, Larouche R. Pharmacokinetics and comparative bioavailability of a levothyroxine sodium oral solution and soft capsule. Clin Pharmacol Drug Dev. 2019;8:521-8.

2. Shah HS, Chaturvedi K, Hamad M, Bates S, Hussain A, Morris K. New insights on solid-state changes in the levothyroxine sodium pentahydrate during dehydration and its relationship to chemical instability. AAPS PharmSciTech. 2019;20:39.

3. Iovino M, Iovine N, Petrosino A, Giagulli VA, Licchelli B, Guastamacchia E, et al. Sevelamer carbonate markedly reduces levothyroxine absorption. Endocr Metab Immune Disord Drug Targets. 2014;14:206-9.

4. Parizi MPS, Lastre Acosta AM, Ishiki HM, Rossi RC, Mafra RC, Teixeira ACSC. Environmental photochemical fate and UVC degradation of sodium levothyroxine in aqueous medium. Environ Sci Pollut Res. 2019;26:4393-403.

5. Shah RB, Collier JS, Sayeed VA, Bryant A, Habib MJ, Khan MA. Tablet splitting of a narrow therapeutic index drug: a case with levothyroxine sodium. AAPS PharmSciTech. 2010;11:1359-67.

6. Guglielmi R, Grimaldi F, Negro R, Frasoldati A, Misischi I, Graziano F, et al. Shift from levothyroxine tablets to liquid formulation at breakfast improves quality of life of hypothyroid patients. Endocr Metab Immune Disord Drug Targets. 2018;18:235-40.

7. Negro R. Thyroid and assisted reproduction technologies: a brief clinical update with recommendations for practice. Endocr Metab Immune Disord Drug Targets. 2018;18:194-200.

8. Whitney KE, Ackerman KE, Tenforde AS. Chapter 15 - Bone health of the runner: metabolic workup and impact on fracture risk. In: Harrast MA, editor. Clinical Care of the Runner. Amsterdam: Elsevier; 2020. pp. 153-68.

9. Ayoub MM, Jasti B, Elantouny NG, Elnahas H, Ghazy F-E. Comparative study of PLGA in-situ implant and nanoparticle formulations of Entecavir; in-vitro and in-vivo evaluation. J Drug Deliv Sci Technol. Elsevier; 2020;56:101585.

10. Astaneh R, Moghimi HR, Erfan M, Mobedi H. Formulation of an injectable implant for peptide delivery and mechanistic study of the effect of polymer molecular weight on its release behavior. Daru. 2006; 14:65-70.

11. Kamali H, Khodaverdi E, Hadizadeh F, Yazdian-Robati R, Haghbin A, Zohuri G. An in-situ forming implant formulation of naltrexone with minimum initial burst release using mixture of PLGA copolymers and ethyl heptanoate as an additive: in-vitro, ex-vivo, and in-vivo release evaluation. J Drug Deliv Sci Technol. 2018;47:95-105.

12. Graves RA, Freeman T, Mandal TK. In vitro dissolution method for evaluation of buprenorphine in situ gel formulation: a technical note. AAPS PharmSciTech. 2007;8:3-6.

13. Koocheki S, Madaeni SS, Niroomandi P. Development of an enhanced formulation for delivering sustained release of buprenorphine hydrochloride. Saudi Pharm J. 2011;19:255-62.

14. Liu H, Venkatraman SS. Cosolvent effects on the drug release and depot swelling in injectable in situ depot-forming systems. J Pharm Sci. 2012;101:1783-93.

15. Yehia SA, Halim SAA, Aziz MY. Polymeric and non polymeric injectable in-situ forming implant systems for sustained delivery of lornoxicam: in vitro and in vivo evaluation. Curr Drug Deliv. 2018;15:1193-203.

16. Mashayekhi R, Mobedi H, Najafi J, Enayati M. In-vitro/in-vivo comparison of leuprolide acetate release from an in-situ forming plga system. Daru, J Pharm Sci. 2013;21:1.

17. Roberge C, Cros JM, Serindoux J, Cagnon ME, Samuel R, Vrlinic T, et al. BEPO®: bioresorbable diblock mPEG-PDLLA and triblock PDLLA-PEG-PDLLA based in situ forming depots with flexible drug delivery kinetics modulation. J Controlled Rel. 2020;319:416-27.

18. Pilon D, Tandon N, Lafeuille MH, Kamstra R, Emond B, Lefebvre $\mathrm{P}$, et al. Treatment patterns, health care resource utilization, and spending in medicaid beneficiaries initiating second-generation long-acting injectable agents versus oral atypical antipsychotics. Clin Therapeut. 2017;39:1972-85.

19. Kaplan G, Casoy J, Zummo J. Impact of long-acting injectable antipsychotics on medication adherence and clinical, functional, and economic outcomes of schizophrenia. Patient Prefer Adherence. 2013;7:1171.

20. Feng L, Ward Jonette A, Li SK, Tolia G, Hao J, Choo DI. Assessment of PLGA-PEG-PLGA copolymer hydrogel for sustained drug delivery in the ear. Curr Drug Deliv. 2015;11: 279-86.

21. Kamali H, Khodaverdi E, Hadizadeh F, Mohajeri SA. In-vitro, exvivo, and in-vivo evaluation of buprenorphine $\mathrm{HCl}$ release from an in situ forming gel of PLGA-PEG-PLGA using N-methyl-2pyrrolidone as solvent. Mater Sci Eng C. 2019;96:561-75.

22. Ruel-Gariepy E, Leroux J-C. In situ-forming hydrogels-review of temperature-sensitive systems. Eur J Pharm Biopharm. 2004;58: 409-26.

23. Khodaverdi E, Tekie FSM, Mohajeri SA, Ganji F, Zohuri G, Hadizadeh F. Preparation and investigation of sustained drug delivery systems using an injectable, thermosensitive, in situ forming hydrogel composed of PLGA-PEG-PLGA. AAPS PharmSciTech. 2012;13:590-600.

24. Kamali H, Khodaverdi E, Hadizadeh F, Mohajeri SA, Nazari A, Jafarian AH. Comparison of in-situ forming composite using PLGA-PEG-PLGA with in-situ forming implant using PLGA: invitro, ex-vivo, and in-vivo evaluation of naltrexone release. J Drug Deliv Sci Technol. 2019;50:188-200.

25. Zhang C, Liao L, Gong S. Recent developments in microwaveassisted polymerization with a focus on ring-opening polymerization. Green Chem. 2007;9:303-14.

26. Gou M, Gong C, Zhang J, Wang X, Wang X, Gu Y, et al. Polymeric matrix for drug delivery: honokiol-loaded PCL-PEGPCL nanoparticles in PEG-PCL-PEG thermosensitive hydrogel. J Biomed Mater Res A. 2010;93:219-26.

27. Tamboli V, Gyan P, Mishra AKM. Novel pentablock copolymer (PLA-PCL-PEG-PCL-PLA) based nanoparticles for controlled drug delivery: effect of copolymer compositions on the crystallinity of copolymers and in vitro drug release profile from nanoparticles. Colloid Polym Sci. 2013;291:1235-45. 
28. Khodaverdi E, Akbari A, Tekie FSM, Mohajeri SA, Zohuri G, Hadizadeh F. Sustained delivery of amphotericin B and vancomycin hydrochloride by an injectable thermogelling tri-block copolymer. PDA J Pharm Sci Technol. 2013;67:135-45.

29. Gong CY, Shi S, Dong PW, Yang B, Qi XR, Guo G, et al. Biodegradable in situ gel-forming controlled drug delivery system based on thermosensitive PCL-PEG-PCL hydrogel: part 1: synthesis, characterization, and acute toxicity evaluation. J Pharm Sci. 2009;98:4684-94.

30. Payyappilly S, Dhara S, Chattopadhyay S. Thermoresponsive biodegradable PEG-PCL-PEG based injectable hydrogel for pulsatile insulin delivery. J Biomed Mater Res A. 2014;102:1500-9.

31. Zentner GM, Rathi R, Shih C, McRea JC, Seo MH, Oh H, et al. Biodegradable block copolymers for delivery of proteins and waterinsoluble drugs. J Control Release. 2001;72:203-15.

32. Qiao M, Chen D, Ma X, Liu Y. Injectable biodegradable temperature-responsive PLGA-PEG-PLGA copolymers: synthesis and effect of copolymer composition on the drug release from the copolymer-based hydrogels. Int J Pharm Netherlands. 2005;294: 103-12.

33. Bode C, Kranz H, Kruszka A, Siepmann F, Siepmann J. In-situ forming PLGA implants: how additives affect swelling and drug release. J Drug Deliv Sci Technol. 2019;53:101180.

34. Lizambard M, Menu T, Fossart M, Bassand C, Agossa K, Huck O, et al. In-situ forming implants for the treatment of periodontal diseases: simultaneous controlled release of an antiseptic and an antiinflammatory drug. Int J Pharm. 2019;572:118833.

35. Do MP, Neut C, Metz H, Delcourt E, Siepmann J, Mäder K, et al. Mechanistic analysis of PLGA/HPMC-based in-situ forming implants for periodontitis treatment. Eur J Pharm Biopharm. 2015;94:273-83.

36. Bode C, Kranz H, Siepmann F, Siepmann J. In-situ forming PLGA implants for intraocular dexamethasone delivery. Int J Pharm. 2018;548:337-48

37. Shapourgan M, Mobedi H, Sheikh N, Behnamghader A, Mashak A. Leuprolide acetate release study from gamma-irradiated PLGAbased in situ forming system. Curr Drug Deliv. 2017;14:1170-7.

38. Bakhshi R, Vasheghani-Farahani E, Mobedi H, Jamshidi A. Mazyar Khakpour. The effect of additives on naltrexone hydrochloride release and solvent removal rate from an injectable in situ forming PLGA implant. Polym Adv Technol. 2006;17:354-9.

39. Rahimi M, Mobedi $\mathrm{H}$, Behnamghader A. In situ forming poly(lactic acid-co-glycolic acid) implants containing leuprolide acetate/ $\beta$ cyclodextrin complexes: preparation, characterization, and in vitro drug release. Int J Polym Mat Polym Biomat. 2016;65:75-84.

40. Yousefi G, Tamaddon AEP. Synthesis of PEG-PLGA copolymer for preparation of docetaxel nanoparticles. Res Pharm Sci. 2012;7: 2012.
41. Fekri Aval S, Akbarzadeh A, Yamchi MR, Zarghami F, NejatiKoshki K, Zarghami N. Gene silencing effect of SiRNA-magnetic modified with biodegradable copolymer nanoparticles on hTERT gene expression in lung cancer cell line. Artif cells Nanomed, Biotechnol. 2016;44:188-93.

42. Ni X, Cheng A, Li J. Supramolecular hydrogels based on selfassembly between PEO-PPO-PEO triblock copolymers and $\alpha$-cyclodextrin. J Biomed Mater Res - Part A. 2009;88:1031-6.

43. Ahmed TA, Ibrahim HM, Samy AM, Kaseem A, Nutan MTH, Hussain MD. Biodegradable injectable in situ implants and microparticles for sustained release of montelukast: in vitro release, pharmacokinetics, and stability. AAPS PharmSciTech. 2014;15:77280 .

44. Astaneh R, Erfan M, Moghimi H, Mobedi H. Changes in morphology of in situ forming PLGA implant prepared by different polymer molecular weight and its effect on release behavior. J Pharm Sci. 2009;98:135-45.

45. Rhee JH. Current and new approaches for mucosal vaccine delivery. Mucosal Vaccines Innov. Prev. Infect. Dis. Elsevier Inc.; Chapter 19, 2020 325-356.

46. Zare M, Mobedi H, Barzin J, Mivehchi H, Jamshidi A, Mashayekhi R. Effect of additives on release profile of leuprolide acetate in an in situ forming controlled-release system: in vitro study. J Appl Polym Sci. 2007;107:3781-7.

47. Khodaverdi E, Tafaghodi M, Ganji F, Abnoos K, Naghizadeh H. In vitro insulin release from thermosensitive chitosan hydrogel. AAPS PharmSciTech. 2012;13:460-6.

48. Khodaverdi E, Rajabi O, Farhadi F, Jalali A, Tekie FSM. Preparation and investigation of poly (N-isopropylacrylamide-acrylamide) membranes in temperature responsive drug delivery. Iran J Basic Med Sci. 2010;13:102-10.

49. Hines DJ, Kaplan DL. Poly(lactic-co-glycolic) acid-controlledrelease systems: experimental and modeling insights. Crit Rev Ther Drug Carrier Syst. 2013;30:257-76.

50. Lu Y, Wu F, Duan W, Mu X, Fang S, Lu N, et al. Engineering a "PEG-g-PEI/DNA nanoparticle-in- PLGA microsphere" hybrid controlled release system to enhance immunogenicity of DNA vaccine. Mater Sci Eng C. 2020;106:110294.

51. Anversa Dimer F, de Souza Carvalho-Wodarz C, Goes A, Cirnski K, Herrmann J, Schmitt V, et al. PLGA nanocapsules improve the delivery of clarithromycin to kill intracellular Staphylococcus aureus and Mycobacterium abscessus. Nanomedicine. 2020;24:102125.

Publisher's Note Springer Nature remains neutral with regard to jurisdictional claims in published maps and institutional affiliations. 